\title{
Cuidados mixtos y cuidados informales a los mayores dependientes, ¿son complementarios o sustitutivos?: una visión comparada entre los países del sur de Europa ${ }^{1}$
}

$M^{\mathrm{a}}$ Ángeles Minguela Recover

Instituto de Desarrollo Regional, Universidad de Granada

\section{José A. Camacho Ballesta}

Instituto de Desarrollo Regional, Universidad de Granada

<jcamacho@ugr.es>

Espainia, Italia eta Portugalen, familia eta laguntzasare informalak dira zaintzen alorreko hornitzaile nagusiak. Familiaren egituretan eta dinamiketan izaten diren gizarte-aldaketen ondorioz, zaintzaren hornitzaile formalek betetzen dituzte eremu pribatuko hainbat zeregin. Mendekotasuna duten 65 urtetik gorako adinekoek beren etxean hartzen duten zaintza mistoa zer faktorek mugatzen duten aztertzea da gure lanaren helburua. Horretarako, 2011. urteko Survey of Health, Ageing and Retirement in Europe (SHARE) erabili da, laugarren edizioa hain zuzen ere. Etxearen ingurunean egiten diren zaintzak direnez gero, protagonismo handia dute tamainak eta gizarte-sareko kideen arteko hurbiltasunak, zaintzaile informalen osagarri bihurtzen baitira; horrez gainera, estrategia bat dira pertsona bat bere ingurunean mantentzeko.

\section{GAKO-HITZAK:}

Generoa, mendekotasuna, zaintza informalak, zaintza mistoak, hurbiltasun geografikoa.
En España, Italia y Portugal, la familia y la red de apoyos informales son los principales proveedores de cuidados. Los cambios sociales en las estructuras y dinámicas familiares hacen que determinadas tareas realizadas en la esfera privada pasen a ser cubiertas por proveedores formales de cuidado. El objetivo de nuestro trabajo es analizar los factores que determinan la recepción de cuidados mixtos entre los dependientes mayores de 65 años en su domicilio. Para ello, se ha utilizado la Survey of Health, Ageing and Retirement in Europe (SHARE) en su cuarta edición, correspondiente a 2011. Al tratarse de cuidados en el entorno del hogar, el tamaño y la proximidad entre los miembros de la red social adquieren un gran protagonismo, porque se transforman en un complemento para los cuidadores informales, además de ser una estrategia para mantener a la persona en su entorno.

\section{Palabras Clave:}

Género, dependencia, cuidados informales, cuidados mixtos, proximidad geográfica.

${ }^{1}$ Una versión previa de este trabajo se presentó en el V Congreso de la Red Española de Política Social (REPS) 'Desigualdad y democracia: política públicas e innovación social', celebrado el 5 y 6 de febrero de 2015 en Barcelona. 


\section{Introducción}

La proporción de personas mayores de 65 años en los países de sur de Europa rozará el $30 \%$ en 2030 (Eurostat, 2014a). El incremento de la esperanza de vida y la mejora en las condiciones y estilos de vida aumentan el peso de la población mayor dentro de la pirámide demográfica, debido al descenso de las tasas de mortalidad. Simultáneamente, el incremento de la participación de la mujer en el mercado de trabajo, el aumento de los divorcios y los cambios en las estructuras y dinámicas familiares se traducen en el descenso de las tasas de fertilidad. El envejecimiento no sólo provoca una serie de cambios demográficos y sociales, sino que es un desafío para los gobernantes dar respuesta a esta demanda social creciente: la atención a personas en situación de dependencia.

Las situaciones de dependencia son entendidas como una falta o pérdida de autonomía personal -física, psicológica y social- que requiere ayuda de intensidad diferente para el desarrollo de las actividades básicas de la vida cotidiana (Consejo de Europa, 1998). Estas actividades se dividen dos grupos: las actividades básicas de la vida diaria (ABVD) tienen como objetivo el cuidado de uno mismo, por ejemplo, actividades de aseo personal, como lavarse o vestirse; y las actividades instrumentales de la vida diaria (AIVD) son más complejas y generalmente implican una relación de la persona con su entorno, por ejemplo, el manejo del dinero, hacer la compra, las tareas del hogar o ir al médico (Imserso, 2005).

Estas dificultades generan unas necesidades de cuidados personales que pueden ser informales, formales o mixtos. Los cuidados informales suponen la intervención de los miembros de la familia, o de amigos o vecinos del entorno más cercano a la persona en situación de dependencia. Este tipo de cuidado se lleva a cabo normalmente desde la voluntariedad, la generosidad y la solidaridad intergeneracional (Attias-Donfut, 1992; Rodríguez-Rodríguez, 2005). El cuidado formal se ha definido como una atención profesional que requiere unos conocimientos, una formación específica para desarrollar los cuidados, que son remunerados. Los principales proveedores son organizaciones públicas, privadas o instituciones sin ánimo de lucro (Kalwij, Pasini y Wu, 2014). En tercer lugar, los cuidados mixtos son una combinación entre los cuidados informales y los recursos y servicios profesionales prestados por entidades públicas o privadas. Éstos se basan en un sistema complejo que combina la actuación de la familia con el mercado y el Estado (Le Bihan y Martin, 2010).

El campo de estudio relacionado con los cuidados personales es muy extenso y diverso. Entre los estudios recientes centrados en la distribución del cuidado formal e informal en Europa, destacan Motel-Klingebiel, Tesch-Roemer y Von Kondratowitz (2005); Van Groenou et al. (2006); Pommer, Woittiez y Stevens (2007); Bolin, Lindgren y Lundborg (2008); y Lowenstein y Daatland (2006). En ellos se reconoce cómo en los países escandinavos, Bélgica y los
Países Bajos, el cuidado en el hogar es básicamente de carácter formal, combinado o no con cuidados informales. A través de una intensa red de servicios y un sistema de políticas de conciliación de la vida personal y personal con el empleo, responden a la disminución de las redes familiares y al aumento de las personas mayores en situación de dependencia (Pavolini y Ranci, 2008). Por el contrario, en los países mediterráneos, como España, Grecia, Italia y Portugal, la familia tiene un papel protagonista en la prestación de cuidados personales, y dentro de ésta, la mujer. En cambio, la actuación del Estado es de carácter subsidiario y complementa las actuaciones de entidades sin ánimo de lucro y religiosas (Carrera et al., 2013). Es importante resaltar que todos los regímenes de bienestar tienen en cuenta los cuidados familiares como núcleo central de referencia: la denominada esfera familiar del régimen de bienestar (Rodríguez-Cabrero, 2011).

Los estudios no sólo analizan sobre quién -Estado o familia- ha de recaer la responsabilidad de la prestación de cuidados, sino que también, en la construcción de modelos teóricos que explican la relación entre el cuidado formal e informal a partir de las tareas que cada uno desempeña (Travis, 1995; Lyons y Zarit, 1999; Geerts y Van den Bosch, 2012), identifican tres modelos:

- El modelo compensador jerárquico determina la existencia de un orden en la elección de la tipología de cuidado. La primera opción son los cuidados informales, procurados por el cónyuge, luego por sus hijos, otros miembros de la familia y, por último, por los cuidadores formales (Cantor, 1975, 1979). Ante la imposibilidad de los familiares o la severidad de la situación de dependencia, son los cuidados formales los que compensan la prestación de cuidados personales.

- El modelo de especialización de las tareas plantea que el cuidado formal aparece cuando las tareas demandadas exceden las capacidades de los cuidadores informales. Los cuidadores informales desempeñan aquellas tareas que no implican una gran especialización o son muy sencillas, mientras que los cuidados formales son los encargados de realizar aquellas tareas complejas que requieren una formación y especialización. Los proveedores formales e informales proporcionan diferentes tipos de cuidado, en función de sus características y la complejidad de las necesidades del dependiente (Noelker y Bass, 1989; Litwak, Messeri y Silverstein, 1990).

- El modelo de cuidado suplementario (Edelman y Hughes, 1990; Travis, 1995) reconoce que el cuidado formal sustituye al cuidado realizado por los cuidadores informales ante su ausencia temporal o circunstancial (Rogero-García, 2009). Las tareas realizadas por ambos son las mismas, a diferencia de lo que sucede en el modelo complementario (VanHoutven y Norton, 2004; Gannon y Davin, 2010). 
Este trabajo trata de explicar la existencia de un patrón de cuidados mixtos entre los mayores dependientes de 65 años que reciben cuidados personales en su domicilio en España y en el conjunto de países del sur de Europa disponibles en la cuarta edición de la encuesta SHARE. El artículo se estructura, tras esta breve introducción, en tres apartados. En primer lugar, se describe el proceso metodológico de construcción de la muestra, los factores implicados en el trabajo y los modelos estadísticos utilizados. En segundo lugar, se exponen los resultados obtenidos del análisis descriptivo de la situación de los cuidados personales, en general, y de los factores predictivos de los cuidados mixtos en España y en los países del sur de Europa. Y en tercer lugar, se presentan las conclusiones.

\section{Muestra y métodos}

\subsection{La muestra}

La base de datos utilizada para la realización del trabajo es la Survey of Health, Ageing and Retirement in Europe en su cuarta edición (2013a). Los países objeto de estudio son España y los países de sur de Europa disponibles en la encuesta -Italia y Portugal-. Los encuestados son mayores de 65 años, presentan al menos una dificultad en las actividades básicas e instrumentales de la vida diaria (Cuadro 1) y reciben cuidados personales.

\begin{tabular}{|c|c|}
\hline \multicolumn{2}{|c|}{$\begin{array}{l}\text { Cuadro 1. Clasificación de las actividades básicas e } \\
\text { instrumentales de la vida diaria }\end{array}$} \\
\hline Dimensiones & Actividades \\
\hline $\begin{array}{l}\text { Actividades básicas } \\
\text { de la vida diaria } \\
\text { (ABVD): no- de } \\
\text { limitaciones entre } \\
\text { o y } 6\end{array}$ & $\begin{array}{l}\text { - Vestirse o desvestirse. } \\
\text { - Desplazarse dentro de la habitación. } \\
\text { - Comer. } \\
\text { - Cambiar de postura dentro de la cama. } \\
\text { - Usar el baño. } \\
\text { - Levantarse o acostarse en la cama. }\end{array}$ \\
\hline $\begin{array}{l}\text { Actividades } \\
\text { instrumentales de la } \\
\text { vida diaria (AIVD): } n^{0} \\
\text { de limitaciones entre } \\
\text { o y } 7\end{array}$ & $\begin{array}{l}\text { - Usar un mapa para orientarse en un } \\
\text { lugar desconocido. } \\
\text { - Preparar comidas. } \\
\text { - Hacer la compra. } \\
\text { - Llamar por teléfono. } \\
\text { - Administrar medicamentos. } \\
\text { - Hacer tareas de la casa o el jardín. } \\
\text { - Administrar el dinero. }\end{array}$ \\
\hline
\end{tabular}

Fuente: SHARE-Project (2013b).

Para determinar el número de encuestados que reciben cuidados personales, la encuesta plantea las siguientes preguntas: "en los últimos doce meses, ¿algún miembro fuera del hogar, algún amigo o vecino le ha proporcionado cuidado o ayuda en su hogar?; ¿alguien que conviva en su hogar le ha proporcionado ayuda o cuidados personales de manera regular en los últimos doce meses?". Ello permite clasificar a los encuestados que reciben cuidados personales y la tipología de cuidado, atendiendo a los proveedores de cuidados. La muestra resultante es de 445 casos para el conjunto de países del sur de Europa disponibles en la encuesta - Italia y Portugal-, y de otros 299 para España.

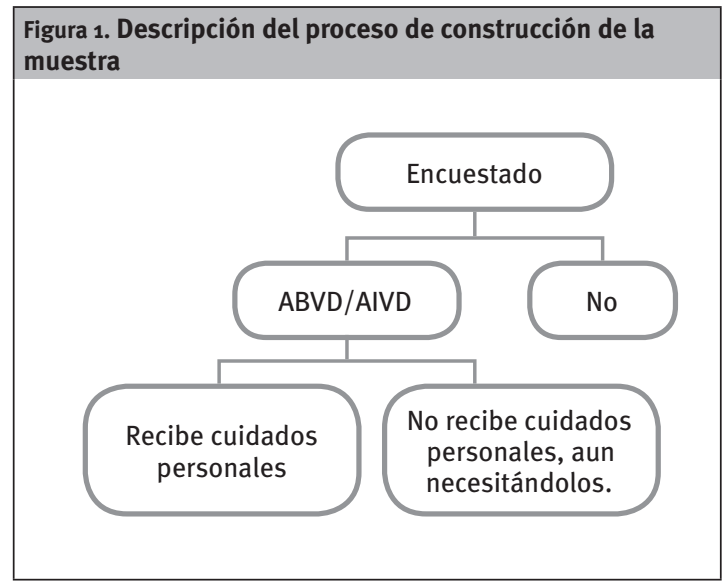

ABVD: actividades básicas de la vida diaria. AIVD: actividades instrumentales de la vida diaria.

Fuente: Elaboración propia.

Los siguientes apartados describen la construcción de las variables que han determinado los factores que intervienen en el trabajo.

\subsection{La variable dependiente: 'cuidados mixtos'}

La encuesta no sólo nos permite conocer el número de encuestados que reciben cuidados personales, sino además identificar quién le proporciona dicho cuidado. De hecho, se identifican los siguientes cuidadores: esposa/pareja, exesposa/expareja, hermanos/as, hijos/as, yernos/nueras, nietos/as, sobrinas/os, otros familiares, (ex)compañeros/as de trabajo y vecinos/as, la administración pública, una organización religiosa, terapeutas u otra ayuda profesional, empleados del hogar o proveedores de atención sanitaria. Se incluyen además los miembros de la red social del entrevistado ${ }^{2}$.

Los cuidados mixtos son, por tanto, aquellos en los que en la prestación del cuidado personal intervienen tanto cuidadores formales ${ }^{3}$ como informales, siendo ésta nuestra variable dependiente, cuyo fin es analizar cuáles son los factores que predicen este tipo de cuidados respecto a los que sólo reciben cuidados informales.

${ }^{2}$ Los datos sobre la red social y su codificación desde 101 al 107 se obtuvieron a través de un generador de nombres (más detalles en Litwin et al., 2013). De ahí que el tamaño de la red social tenga un máximo de 7 miembros.

${ }^{3}$ Los encuestados que sólo reciben cuidados formales no se han tenido en cuenta en nuestro trabajo. 


\subsection{Factores}

\subsubsection{Factores centrados en la persona}

En primer lugar, las características de receptor de los cuidados personales que se toman en cuenta son: el sexo, la edad (65 a 74 años, de 75 a 84 y más de 84 años), el grado de severidad de la situación de dependencia 4 (moderada, severa y total) y la variable dicotómica 'vivir en pareja'. En segundo lugar, las características del hogar son el tamaño (una, dos, o más personas), la variable dicotómica 'tener hijos' que formen parte de la red social del mayor dependiente, los ingresos mensuales del conjunto de los miembros del hogar (menos de $750 € /$ mes, entre 750,01 y $1.300,00 € /$ mes, y más de $1.300 € /$ mes), y si recibe o no prestaciones económicas públicas periódicas, como pensiones de jubilación, jubilación anticipada, subsidio de enfermedad, pensiones de invalidez y prestaciones de asistencia social.

\subsubsection{Factor socioterritorial: la proximidad espacial media con su red social}

La cuarta edición de la encuesta SHARE introduce el módulo social network para caracterizar la red de apoyos existentes alrededor de las personas mayores en Europa y profundizar en su análisis. En el campo de estudio de los cuidados personales, es importante saber quiénes y cuántas personas son las encargadas de prestar dicho cuidado.

En nuestro estudio, necesitábamos un indicador socioterritorial en el que pudiésemos combinar información sobre: a) la distancia física existente entre los miembros de la red social y el encuestado; $y$ b) el tamaño de su red social. La primera parte del indicador consistía en conocer dónde vive cada miembro de la red social respecto al mayor dependiente, información incluida en el módulo social network, en el que encontramos siete variables que codifican las distancias (Tabla 1).

Para abordar la segunda parte de nuestro indicador, partimos de la premisa del marcado carácter subjetivo que tiene la composición de la red social. Ésta está configurada en función de lo importante que para el encuestado sea su relación con determinadas personas de su entorno social, hasta un máximo de siete. El indicador que respondió a nuestras premisas iniciales fue la proximidad espacial media:

Proximidad espacial media $=s n 0061, \ldots$ ,snoo6_7Tamaño de la red social

${ }^{4}$ La encuesta SHARE permite determinar al conjunto de dependientes a través de trece actividades agrupadas en actividades básicas e instrumentales de la vida diaria (Tabla 1), pero no el grado de severidad. Éste se ha calculado atendiendo al número de dificultades que presenta la persona dependiente, y las categorías resultantes son: gravedad moderada (aquellos tienen entre una y cuatro dificultades), gravedad severa (entre cinco y diez dificultades) y gravedad total (más de diez dificultades).
Tabla 1. Codificación de la variable 'proximidad geográfica'

\begin{tabular}{|l|c|}
\hline Categoría & Codificación \\
\hline Mismo hogar & 1 \\
\hline Mismo edificio & 2 \\
\hline <1 km & 3 \\
\hline $1-5 \mathrm{~km}$ & 4 \\
\hline $5-25 \mathrm{~km}$ & 5 \\
\hline $25-100 \mathrm{~km}$ & 6 \\
\hline $100-500 \mathrm{~km}$ & 7 \\
\hline >500 km & 8 \\
\hline Específica país & 9 \\
\hline No consta* & 0 \\
\hline
\end{tabular}

* Nueva categoría, no incluida en la codificación original. Fuente: Elaboración propia.

Tabla 2. Codificación de la nueva variable 'proximidad geográfica media'

\begin{tabular}{|l|c|c|}
\hline Categorías & Valores medios & Codificación \\
\hline Mismo hogar & $1-2,86$ & 1 \\
\hline$<1 \mathrm{~km}$ & $3-3,86$ & 2 \\
\hline $1-5 \mathrm{~km}$ & $4-4,86$ & 3 \\
\hline$>5 \mathrm{~km}$ & $5-9$ & 4 \\
\hline No consta & 0 & 5 \\
\hline
\end{tabular}

Fuente: Elaboración propia.

Los valores resultantes del indicador oscilaban entre o y 9. Los valores o se transformaron en una nueva categoría 'no consta'. Por lo tanto, el nuevo rango de valores estaba comprendido entre 1 y 9. La Tabla 1 muestra el proceso de codificación de la nueva variable 'proximidad espacial media' y las categorías resultantes, atendiendo a los valores medios y tomando como referencia las categorías establecidas por la variable 'proximidad' para los valores enteros.

Como resultado, se obtiene la nueva variable 'proximidad geográfica media' que tiene en cuenta a todos los miembros de la red social y la distancia existente entre ellos, a través de sus valores medios.

\subsection{Análisis: regresión logística binaria}

El análisis estadístico consistió en realizar tablas de contingencia, aplicando pruebas de correlación y $\chi^{2}$ cuadrado para comprobar la asociación de las variables implicadas en el estudio. Asimismo, se realizaron dos modelos comparativos de regresión logística binaria hacia delante: uno formado por Italia y Portugal, y otro compuesto exclusivamente por España. El uso de este método estadístico pretendía explorar la probabilidad que tienen las personas dependientes de recibir cuidados mixtos, en función de los factores (variables independientes) implicados en el trabajo. A través de las razón de posibilidades $^{5}$ (en adelante, $R P$ ) se comprobó la probabilidad

${ }^{5}$ La razón de posibilidades es la traducción del término estadístico inglés odds ratio (Tapia y Nieto, 1993). 
de cambio en la variable predictora respecto a la categoría de referencia. Además, se utilizaron como medidas de bondad de ajuste una versión corregida de la $R^{2}$ de Cox-Snell, la $R^{2}$ de Nagelkerke. En nuestro trabajo, la $R^{2}$ de Nagelkerke reflejó un buen ajuste del modelo: 0,125 para los países de sur de Europa y 0,261 para España. Para los cálculos estadísticos, se utilizó el programa IBM SPSS versión 20.0.

\section{Resultados}

\subsection{Resultados descriptivos}

Los datos descriptivos nos proporcionan la radiografía de los dependientes mayores de 65 años que reciben cuidados personales para un conjunto de variables recogidas en la Tabla 2, en España y los países del sur de Europa. En torno al $15 \%$ de los encuestados que reciben cuidados personales son mixtos, siendo el resto cuidados informales. Desde el punto de vista de género, las mujeres son la que reciben el 65,5\% de los cuidados en el sur de Europa, aunque en España esa cifra es aún mayor. Es común para España y los países del sur de Europa que los cuidados presenten altos porcentajes en el tramo de edad de los 75 a los 84 años, coincidiendo con los valores de la esperanza de vida, que, en ambos casos, están en torno a los 80 años (Eurostat, 2014b). Sin embargo, el envejecimiento dentro de la muestra española hace que aproximadamente el 30,0\% de los cuidados son recibidos por mayores de 84 años.

La pérdida de autonomía personal para la realización de las actividades de la vida cotidiana implica un agravamiento de la situación de dependencia y un incremento de los cuidados personales. En la Tabla 2, observamos cómo en los países del sur de Europa el 34,2\% de los cuidados personales los reciben aquellos que tienen un grado de dependencia severa, siendo esa cifra menor para España. En cambio, los cuidados para los encuestados con un grado de severidad total representan el $46,5 \%$ en España, porcentaje ligeramente superior al de los países del sur de Europa. Aunque la atención está correlacionada con la necesidad de las personas, en España la intensidad es mayor respecto al conjunto de los países del sur estudiados.

Las personas dependientes de nuestra muestra viven en su entorno social habitual y los cuidados personales los reciben en su domicilio. Por lo tanto, el tamaño del hogar del dependiente es el núcleo donde se desempeñan las principales tareas asociadas al cuidado personal. De esta manera, los hogares unipersonales de los países del sur de Europa reciben el 35,5\% de los cuidados personales. Tanto en los países del sur de Europa como en España, los hogares formados por dos miembros son los que mayores porcentajes de cuidados reciben. Esta afirmación se justifica porque más de la mitad de los dependientes conviven con sus parejas, siendo éstas sus proveedoras potenciales de cuidados personales.
En España, el $70 \%$, y en los países del sur de Europa, el $64,5 \%$ de los mayores dependientes que reciben cuidados personales implican a los hijos adultos, que forman parte de su red social en ese cuidado. La función de éstos está dirigida a complementar tanto las acciones realizadas por otros miembros -pareja, otros hijos o familiares-como los apoyos formales que se estén recibiendo; también puede representar una dedicación en exclusiva al cuidado personal del familiar dependiente cuando viven en el mismo domicilio.

De esta manera, la perspectiva territorial implica que la proximidad geográfica media entre la persona dependiente y los miembros de su red social siga un patrón similar tanto en España como en los países del sur de Europa. En alrededor del 65-70\% del total de los mayores que reciben cuidados personales, la distancia es inferior a un kilómetro entre los miembros de su red social. Esto facilita el control y la supervisión continuada de la situación de dependencia del mayor. Sin embargo, cuanto mayor es la distancia, menor es el porcentaje de los cuidados que reciben, fenómeno éste más pronunciado en España.

Finalmente, la situación económica en la que se encuentran los mayores dependientes también va a condicionar la tipología de cuidado que reciban. De hecho, la pérdida de poder adquisitivo asociada a la jubilación y las situaciones de pobreza femenina debidas a una limitada cotización a los sistemas de Seguridad Social determinan la realidad social de los mayores dependientes. En este sentido, en los países del sur de Europa el $24 \%$ de los mayores que reciben cuidados tienen una renta mensual en su hogar de más de 1.300 euros; en cambio, en España no alcanzan el $20 \%$ y y el $32 \%$ de los mayores de 65 años tienen una renta mensual inferior a los $750 €$. De esta manera, los sistemas de protección social a través de prestaciones económicas públicas tienden a responder a las necesidades de atención relacionadas con la edad y las situaciones de dependencia. De ahí las diferencias entre España y el resto de países del sur de Europa, donde el $64,3 \%$ de los mayores dependientes que reciben prestaciones económicas públicas.

\subsection{Resultados del análisis de regresión logística binaria}

Los resultados de la regresión logística binaria (Tabla 3) examinan los factores que interviene en la prestación de cuidados mixtos entre los mayores de 65 años dependientes que reciben cuidados informales. En los factores que forman nuestro análisis, encontramos diferencias y semejanzas entre España y los países del sur de Europa. Entre las diferencias, se encuentran que el sexo de la persona dependiente no es un factor representativo para el conjunto de países de sur Europa, pero sí para España. En otras palabras, una mujer española dependiente tiene aproximadamente cuatro veces más probabilidades de recibir cuidados mixtos que un hombre. 
Tabla 3. Personas en situación de dependencia en los países del sur de Europa que reciben cuidados personales en su domicilio

\begin{tabular}{|c|c|c|c|c|c|}
\hline & & $\begin{array}{r}\text { País } \\
\text { de }\end{array}$ & $\begin{array}{l}\text { del sur } \\
\text { ropa }\end{array}$ & & |ก̃a \\
\hline Variable & Categorías & $\mathbf{N}$ & $\%$ & $\mathbf{N}$ & $\%$ \\
\hline & Mixtos & 65 & 14,6 & 47 & 15,7 \\
\hline Tipología de cuidados & Informales & 380 & 85,4 & 252 & 84,3 \\
\hline & Total & 445 & 100,0 & 299 & 100,0 \\
\hline Covariables & Categorías & $\mathbf{N}$ & $\%$ & $\mathbf{N}$ & $\%$ \\
\hline & Hombre & 155 & 34,8 & 89 & 29,8 \\
\hline & Mujer & 290 & 65,2 & 210 & 70,2 \\
\hline & 65-74 años & 157 & 35,3 & 63 & 21,1 \\
\hline Edad & $75-84$ años & 215 & 48,3 & 154 & 51,5 \\
\hline & $\geq 85$ años & 73 & 16,4 & 82 & 27,4 \\
\hline & Moderada & 119 & 26,7 & 69 & 23,1 \\
\hline $\begin{array}{l}\text { Grado de severidad de la } \\
\text { situación de dependencia }\end{array}$ & Severa & 152 & 34,2 & 91 & 30,4 \\
\hline & Total & 174 & 39,1 & 139 & 46,5 \\
\hline & $\geq 3$ miembros & 92 & 20,7 & 74 & 24,7 \\
\hline Tamaño del hogar & 2 miembros & 195 & 43,8 & 136 & 45,5 \\
\hline & 1 miembro & 158 & 35,5 & 89 & 29,8 \\
\hline & No vivir en pareja & 207 & 46,5 & 132 & 44,1 \\
\hline Vivir en pareja & Vivir en pareja & 238 & 53,5 & 167 & 55,9 \\
\hline & No tener hijos en la red social & 158 & 35,5 & 88 & 29,4 \\
\hline & Tener hijos en la red social & 287 & 64,5 & 211 & 70,6 \\
\hline & Mismo hogar & 172 & 38,7 & 118 & 39,5 \\
\hline & $<1 \mathrm{~km}$ & 119 & 26,7 & 109 & 36,5 \\
\hline Proximidad geográfica media & $1-5 \mathrm{~km}$ & 69 & 15,5 & 38 & 12,7 \\
\hline & $>5 \mathrm{~km}$ & 53 & 11,9 & 24 & 8,0 \\
\hline & No consta & 32 & 7,2 & 10 & 3,3 \\
\hline & No recibe ayuda pública & 159 & 35,7 & 156 & 52,2 \\
\hline & Recibe ayuda pública & 286 & 64,3 & 143 & 47,8 \\
\hline & $<750,00 € /$ mes & 120 & 27,0 & 84 & 28,1 \\
\hline $\begin{array}{l}\text { Ingresos mensuales de los } \\
\text { miembros del hogar }\end{array}$ & $750,01-1 \cdot 300,00 € /$ mes & 159 & 35,7 & 105 & 35,1 \\
\hline & $\geq 1.300,01 € /$ mes & 166 & 37,3 & 110 & 36,8 \\
\hline
\end{tabular}

Fuente: Elaboración propia. 
Tabla 4. Modelos de regresión logística de los factores asociados a la recepción de cuidado mixto

\begin{tabular}{|c|c|c|c|c|c|c|c|c|c|c|c|}
\hline \multirow{3}{*}{ Variable } & \multirow{3}{*}{ Categorías } & \multicolumn{5}{|c|}{ España } & \multicolumn{5}{|c|}{ Países del sur de Europa } \\
\hline & & \multirow{2}{*}{ B } & \multirow{2}{*}{ Sig. } & \multirow{2}{*}{$\operatorname{Exp}(B)$} & \multicolumn{2}{|c|}{ I.C. $95 \%$ para EXP(B) } & \multirow{2}{*}{ B } & \multirow{2}{*}{ Sig. } & \multirow{2}{*}{$\operatorname{Exp}(B)$} & \multicolumn{2}{|c|}{ I.C. $95 \%$ para EXP(B) } \\
\hline & & & & & Inferior & Superior & & & & Inferior & Superior \\
\hline \multirow{2}{*}{ Sexo } & Hombre* & & & & & & & & & & \\
\hline & Mujer & 1,33 & 0,02 & 3,79 & 1,27 & 11,26 & 0,19 & 0,57 & 1,21 & 0,63 & 2,32 \\
\hline \multirow{3}{*}{ Edad } & $65-74$ años* & & 0,10 & & & & & 0,79 & & & \\
\hline & $75-84$ años & 0,11 & 0,83 & 1,12 & 0,39 & 3,21 & 0,04 & 0,89 & 1,05 & 0,55 & 1,98 \\
\hline & $\geq 85$ años & 0,92 & 0,11 & 2,51 & 0,82 & 7,68 & $-0,23$ & 0,61 & 0,80 & 0,33 & 1,91 \\
\hline \multirow{3}{*}{$\begin{array}{l}\text { Grado de } \\
\text { severidad de } \\
\text { la situación de } \\
\text { dependencia }\end{array}$} & Moderada* & & 0,01 & & & & & 0,03 & & & \\
\hline & Severa & 1,75 & 0,01 & 5,76 & 1,48 & 22,37 & 0,64 & 0,12 & 1,89 & 0,84 & 4,28 \\
\hline & Total & 1,95 & 0,00 & 7,02 & 1,88 & 26,24 & 1,09 & 0,01 & 2,99 & 1,31 & 6,80 \\
\hline \multirow{3}{*}{$\begin{array}{l}\text { Tamaño del } \\
\text { hogar }\end{array}$} & $\begin{array}{l}\geq 3 \\
\text { miembros* }\end{array}$ & & 0,16 & & & & & 0,01 & & & \\
\hline & 2 miembros & 0,98 & 0,04 & 2,66 & 0,86 & 8,22 & 1,20 & 0,04 & 3,33 & 1,09 & 10,21 \\
\hline & 1 miembro & 1,11 & 0,07 & 3,02 & 0,91 & 10,00 & 1,78 & 0,00 & 5,92 & 1,81 & 19,41 \\
\hline \multirow{5}{*}{$\begin{array}{l}\text { Proximidad } \\
\text { geográfica } \\
\text { media }\end{array}$} & $\begin{array}{l}\text { Mismo } \\
\text { hogar* }\end{array}$ & & 0,18 & & & & & 0,25 & & & \\
\hline & く $1 \mathrm{~km}$ & 0,86 & 0,05 & 2,37 & 1,01 & 5,55 & 0,45 & 0,27 & 1,57 & 0,71 & 3,50 \\
\hline & $1-5 \mathrm{~km}$ & 0,80 & 0,04 & 2,64 & 0,82 & 8,48 & 0,98 & 0,03 & 2,67 & 1,10 & 6,43 \\
\hline & $>5 \mathrm{~km}$ & 1,31 & 0,04 & 3,69 & 1,04 & 13,12 & 0,36 & 0,47 & 1,44 & 0,54 & 3,84 \\
\hline & No consta & 0,47 & 0,60 & 1,60 & 0,28 & 9,25 & 0,72 & 0,19 & 2,05 & 0,70 & 6,01 \\
\hline \multirow{2}{*}{$\begin{array}{l}\text { Prestaciones } \\
\text { económicas } \\
\text { públicas } \\
\text { periódicas }\end{array}$} & $\begin{array}{l}\text { No recibe } \\
\text { ayuda } \\
\text { pública* }\end{array}$ & & & & & & & & & & \\
\hline & $\begin{array}{l}\text { Recibe } \\
\text { ayuda } \\
\text { pública }\end{array}$ & 0,54 & 0,04 & 1,72 & 0,77 & 3,88 & 0,16 & 0,59 & 1,17 & 0,65 & 2,11 \\
\hline \multirow{3}{*}{$\begin{array}{l}\text { Ingresos } \\
\text { mensuales de } \\
\text { los miembros } \\
\text { del hogar }\end{array}$} & $\begin{array}{l}\langle 750,00 € / \\
\text { mes* }^{\star}\end{array}$ & & 0,01 & & & & & 0,41 & & & \\
\hline & $\begin{array}{l}750,01- \\
1.300,00 € / \\
\text { mes }\end{array}$ & $-0,77$ & 0,09 & 0,47 & 0,19 & 1,13 & 0,98 & 0,01 & 1,01 & 0,50 & 2,03 \\
\hline & $\begin{array}{l}\geq 1.300,01 € / \\
\text { mes }\end{array}$ & $-1,50$ & 0,00 & 0,22 & 0,09 & 0,58 & 0,74 & 0,03 & 1,56 & 0,72 & 3,35 \\
\hline \multicolumn{2}{|l|}{ Constante } & $-5,70$ & 0,00 & 0,00 & & & $-4,57$ & 0,00 & 0,01 & & \\
\hline
\end{tabular}

*Categoría de referencia.

Fuente: Elaboración propia.

Los factores económicos marcan también una clara diferencia. En España, aquellos que reciben prestaciones económicas públicas tiene 1,7 veces más probabilidades de recibir cuidados mixtos que aquellos que no las reciben. En cambio, en los países del sur de Europa el acceso a este tipo de cuidados se determina a través de los ingresos mensuales del hogar.

En los hogares con una renta superior a $750 € / \mathrm{mes}$, la probabilidad de recibir cuidados mixtos aumenta: en concreto, cuando la renta supera los 1.300,01 euros, la probabilidad es 1,5 veces superior. Por otro lado, España y los países del sur de Europa muestran como regla la proporcionalidad del grado de dependencia con la probabilidad de recibir cuidados mixtos.

Los encuestados con un grado de severidad total tiene una alta probabilidad (España, $R P=7,02$; y paí- ses del sur de Europa, $R P=2,98$ ) de recibir cuidados mixtos respecto a los clasificados con una dependencia moderada. En cambio, para los países del sur de Europa un grado de dependencia severa no es una categoría representativa a la hora de recibir cuidados mixtos. Sin embargo, en España la probabilidad de recibir cuidados mixtos se incrementa proporcionalmente a la pérdida de autonomía personal o al aumento del grado de severidad del encuestado. Entre las razones que justifican que la edad no es un factor predictivo, se encuentra la asociación con el grado de dependencia, es decir, a mayor edad, menor es la autonomía personal para la realización de las ABVD y las AIVD.

Resulta interesante analizar cómo en países de corte familista la externalización de determinadas tareas relacionadas con la vida diaria del dependiente hace 
que los cuidados mixtos sean un complemento a los cuidados informales. De hecho, los hogares formados por dos personas tienen 2,6 veces más probabilidades de recibir cuidados mixtos que los de tres o más miembros -la probabilidad de cuidados mixtos en estos últimos hogares es mayor en España que en los otros países analizados-. Además esta probabilidad se incrementa en España hasta las 6 veces más cuando los hogares son unipersonales, siendo la mitad $(R P=3,02)$ en los países del sur.

Ahora bien la perspectiva territorial, considerada a través de la distancia física media entre los miembros de la red social y el mayor dependiente, también determina el tipo de cuidado. En nuestro estudio, tomando como categoría de referencia el mismo hogar, se observa que en España cuanto mayor es la distancia física, la probabilidad de recibir cuidados mixtos aumenta. En cambio, en los países del sur de Europa cuando la distancia media está entre 1 y 5 kilómetros la probabilidad de recibir cuidados mixtos es 2,6 veces mayor respecto a los que viven en el mismo hogar.

\section{Conclusiones}

En un contexto social común en el que la prestación de cuidados a familiares dependientes es una obligación legal de la familia, nuestro trabajo presenta un modelo comparativo de los determinantes de recibir cuidados mixtos -formales e informales- entre los dependientes mayores de 65 años en España y el conjunto de países del sur de Europa analizados.

Un alto porcentaje del cuidado que reciben las personas mayores es de naturaleza informal, principalmente llevado a cabo por la familia y los miembros de la red social. De ahí que este tipo de cuidados sean sustitutivos de los cuidados formales cuando la situación de dependencia no es compleja. En cambio, habitualmente los cuidados mixtos son complementarios a medida que aumenta el grado de severidad de la situación de dependencia, intensificándose conforme aumenta la complejidad de las tareas (Bonsang, 2009). La complementariedad de los cuidados mixtos es un factor común tanto para España como para el conjunto de países del sur de Europa estudiados.

En términos de género, las mujeres españolas tienen una mayor probabilidad de recibir cuidados mixtos con respecto a los hombres. Entre las razones que lo justifican, encontramos que las mujeres tienen una mayor esperanza de vida y una mayor probabilidad de vivir solas. Suelen casarse o vivir en pareja con hombres mayores que ellas, y el resultado es que se reduce la probabilidad de que reciban cuidado por parte de sus parejas (Jiménez-Martín y VilaplanaPrieto, 2012; Rodríguez, 2013).

Los cuidados mixtos, a diferencia de los informales, tienen un elevado componente económico, lo que marca diferencias entre España y el conjunto de los países del sur de Europa -Italia y Portugal-.
En el caso de España, los mayores dependientes que perciben prestaciones económicas públicas periódicas tienen mayor probabilidad de recibir cuidados mixtos. En este sentido, se reconoce que la incorporación de la cartera de servicios y prestaciones recogidas en la Ley de Promoción de la Autonomía Personal y Atención a las Personas en Situación de Dependencia (LAPAD) contribuye a la transformación del modelo tradicional de cuidados en los domicilios (Martínez-Buján, 2014). No obstante, la implicación de la familia sigue siendo central y el Estado mantiene su carácter subsidiario.

En los países del sur Europa analizados, a pesar de contar con prestaciones económicas públicas relacionadas con la prestación de cuidado hacia personas dependientes (Da Roit, 2007), en nuestro análisis no se ha presentado como un factor significativo. Por el contrario, es el poder adquisitivo el que determina que el mayor dependiente reciba cuidados mixtos.

El mantenimiento de la persona el mayor tiempo posible en su entorno social habitual refuerza la función de los cuidados mixtos como complemento a los cuidados familiares. De hecho, nuestro estudio demuestra cómo el tamaño del hogar y la proximidad geográfica media entre los miembros de su red social determinan la probabilidad de recibir cuidados mixtos en una doble dirección: por un lado, se profesionaliza el cuidado, el cual, responde a la heterogeneidad y complejidad de las necesidades del dependiente; y por otro, se reduce la carga asociada al cuidador informal más cercano.

Para el conjunto de países del sur de Europa analizados, el cuidado informal es un complemento cuando la distancia está entre 1 y $5 \mathrm{~km}$ e implica un desplazamiento, pero no una supervisión continuada de la situación del mayor dependiente. En cambio, en España el tamaño de la red social con la que cuenta el mayor dependiente hace de la proximidad geográfica un factor determinante a la hora de recibir cuidados mixtos, principalmente, porque el cuidado está formado por un conjunto de rutinas diarias que implican a los miembros del hogar o de la red social. Entonces, con el fin de mejorar la calidad de vida del mayor dependiente, el intercambio de tareas y tiempo es compartido, lo que hace de los cuidados mixtos un respiro para los cuidadores principales.

En síntesis, el incremento de la población mayor y de las situaciones de dependencia aumenta la necesidad de responder a esta demanda social creciente por parte de los gobernantes de los países, máxime en tiempos de crisis y recortes presupuestarios, en los que las percepciones sobre las responsabilidades públicas y privadas respecto a la prestación de cuidados personales es un tema de debate. A pesar de la cercanía con nuestros vecinos del sur de Europa -Italia y Portugal-, podemos resaltar importantes diferencias que se derivan de la instrumentalización de las políticas públicas que afectan a la dependencia. Ello pone de manifiesto la necesidad de tener en cuenta estos factores (redes sociales, familia, proxi- 
midad geográfica, recursos económicos) a la hora de articular las políticas sociales de atención a las personas en situación de dependencia, de forma que los cuidados mixtos sean el símbolo de una sociedad que responde a las necesidades de esta demanda social creciente desde la proximidad.

\section{Agradecimientos}

Este trabajo usa los datos SHARE wave 4 release 1.1.1. publicados el 28 de marzo de 2013 («http:// dx.doi.org/10.6103/SHARE.w4.111〉). La encuesta SHARE ha sido principalmente financiada por la Comisión Europea a través del quinto Programa
Marco (proyecto QLK6-CT-2001-00360, dentro del programa temático Calidad de Vida), a través del 6을 Programa Marco (proyectos SHARE-I3, RII-CT-2006-062193) y a través del 7ํ Programa Marco (SHARE-PREP, $\mathrm{n}^{\circ}$ 211909; SHARE-LEAP, $n^{\circ}$ 227822; y SHARE M4, $n^{\circ}$ 261982). Además, cuenta con la financiación adicional del Instituto Nacional sobre el Envejecimiento (U.S. National Institute on Aging) [U01 AG09740-13S2, AG005842 P01, P01 AG08291, AG12815 P30, R21 AG025169, Y1-AG-455301, IAG BSRo6-11 y OGHA 04-064] y el Ministerio Educación e Investigación alemán, así como de diversas fuentes nacionales (véase 〈http:// www. share-project.org> para obtener una lista completa de las instituciones participantes). 
ATIIAS-DONFUT, C. (1992): “Dependencia de las personas mayores y ayuda intergeneracional", Papers. Revista de Sociología, no 40, págs. 13-33.

BOLIN, K.; LINDGREN, B; y LUNDBORG, P. (2008): “Informal and formal care among single-living elderly in Europe", Health Economics, vol. 17, no 3 , págs. 393-409.

BONSANG, E. (2009): “Does informal care from children to their elderly parents substitute for formal care in Europe?", Journal of Health Economics, vol. $28, \mathrm{n}$ 을 1 págs. 143-154.

CANTOR, M. H. (1979): “Neighbors and friends: An overlook resource in the informal support system", Research on Aging, vol. 1, págs. 434-463.

- (1975): "Life space and the social support system of the inner city elderly of New York", The Gerontologist, vol. 15, págs. 23-27.

CARRERA, F. et al., (2013): "Long-term care systems in comparative perspective: Care needs, informal and formal coverage, and social impacts in European countries", en RANCI, C.; y PAVOLINI, E. (eds.), Reforms in Long-Term Care Policies in Europe, Springer Science+Business Media, Nueva York, págs. 23-52.

CONSEJO DE EUROPA (1998) Recommendation No. $R$ (98) 9 of the Committee of Ministers to Member States on dependence (Adopted by the Committee of Ministers on 18 September 1998 at the $641^{\text {st }}$ meeting of the Ministers' Deputies).

DA ROIT, B. (2007): “Changing intergenerational solidarities within families in a Mediterranean welfare state: Elderly care in Italy", Current Sociology, vol. 55, no 2, págs. 251-269 [khttp://dx.doi. org/10.1177/0011392107073306)].

EDELMAN, P.; y HUGHES, S. (1990): “The impact of community care on provision of informal care to homebound elderly persons", Journal of Gerontology, vol. 45, nํㅡ 2, págs. S74-S84.

EUROSTAT (2014a): “Population projections by sex and single year age" [<http://ec.europa.eu/ eurostat $/ \mathrm{tgm} / \mathrm{table} . \mathrm{do}$ ?tab=table\&init $=1 \& \mathrm{l}$ anguage $=$ en $\&$ pcode $=$ tps00002\&plugin $=1>$, consultado el 9-1-2015].

- (2014b): "Life expectancy at birth, by sex" [ [http:// ec.europa.eu/eurostat/tgm/table.do?tab=table \&init $=1$ \&language $=$ en $\&$ pode $=$ tps00025\&plug in=1>, consultado el 9-1-2015].

GANNON, B.; y DAVIN, B. (2010): "Use of formal and informal care services among older people in Ireland and France", The European Journal of Health Economics, vol. 11, ํㅜ 5, págs. 499-511.

GEERTS, J.; y VAN DEN BOSCH, K. (2012): "Transitions in formal and informal care utilisation amongst older Europeans: The impact of national contexts", European Journal of Ageing, vol. 9, no 1 , págs. 27-37 [<http://dx.doi.org/10.1007/ S10433-011-0199-Z>].

IMSERSO (2005): "Bases demográficas: estimación, características y perfiles de las personas en situación de dependencia”, en IMSERSO, Atención a las personas en situación de dependencia en España. Libro blanco, Madrid, Imserso, págs. 19-9o [<http://www. dependencia.imserso.es/InterPresent2/ groups/imserso/documents/binario/ libroblanco.pdf>].

JIMÉNEZ-MARTÍN, S.; y VILAPLANA-PRIETO, C. (2012): “The trade-off between formal and informal care in Spain", The European Journal of Health Economics, vol. 13, № 4, págs. 461-490.

KALWIJ, A.; PASINI, G.; y WU, M. (2014):“Home care for the elderly: The role of relatives, friends 
and neighbors", Review of Economics of the Household, vol. 12, nㅡㄹ, págs. 379-404.

LE BIHAN, B.; y MARTIN, C. (2010): "Reforming long term care policy in France: Public-private complementarities", Social Policy \& Administration, vol. 44, $\mathrm{n}-4$, págs. 392-410 [<http://dx.doi.org/10.1111 /j.1467-9515.2010.00720.x>].

LITWAK, E.; MESSERI,P.; y SILVERSTEIN, M. (1990): “The role of formal and informal groups in providing help to older people", Marriage \& Family Review, vol. 15, no 1-2, págs. 171-193.

LITWIN, H. et al. (2013): "Social network measurement in SHARE Wave 4", en MALTER, F.; y BÖRSCHSUPAN, A. (eds.), SHARE Wave 4: Innovations \& Methodology, Munich, MEA; Max Planck Institute for Social Law and Social Policy, págs. 18-37.

LOWENSTEIN, A.; y DAATLAND, S. O. (2006): "Filial norms and family support in a comparative crossnational context: Evidence from the OASIS Study", Ageing \& Society, vol. 26, nํ2, págs. 203-223.

LYONS K. S.; y ZARIT, S. H. (1999): "Formal and informal support: The great divide", The International Journal of Geriatric Pysichiatry, vol. 14, $\mathrm{n}^{0} 3$, págs. 183-196.

MARTÍNEZ-BUJÁN, R. (2014): “Los modelos territoriales de organización social del cuidado a personas mayores en los hogares", Revista Española de Investigaciones Sociológicas, vol. 145, págs. 99-126 [<http://dx.doi.org/10.5477/cis/ reis.145.99>].

MOTEL-KLINGEBIEL, A.; TESCH-ROEMER, C.; y VON KONDRATOWITZ, H. (2005): "Welfare states do not crowd out the family: Evidence for mixed responsibility from comparative analyses", Ageing \& Society, vol. 25, n- 6, págs. 863-882.

NOELKER, L. S.; y BASS, D. M. (1989): “Home care for elderly persons: Linkages between formal and informal caregivers", Journal of Gerontology, vol. 44, no 2, págs. S63-S70.

PAVOLINI, E.; y RANCI, C. (2008): “Restructuring the welfare state: Reforms in long-term care in western European countries", Journal of European Social Policy, vol. 18, nํㅜ 3, págs. 246-259 [khttp:// dx.doi.org/10.1177/0958928708091058>].

POMMER E.; WOITTIEZ, I.; y STEVENS, J. (2007): Comparing Care: The Care of the Elderly in Ten EU- countries, La Haya, Netherlands Institute for Social Research/SCP.

RODRÍGUEZ, M. (2013): “Use of informal and formal care among community dwelling dependent elderly in Spain", European Journal of Public Health [<http://dx.doi.org/10.1093/eurpub/ckto88〉].

RODRÍGUEZ-CABRERO, G. (2011): “Políticas sociales de atención a la dependencia en los regímenes de bienestar de la Unión Europa”, Cuadernos de Relaciones Laborales, vol. 29, nㅡ1, págs. 13-42.

RODRÍGUEZ-RODRÍGUEZ, P. (2005): “El apoyo informal a las personas mayores en España y la protección social a la dependencia: del familismo a los derechos de ciudadanía", Revista Española de Geriatría y Gerontología, vol. 40, nํsupl. 2, págs. 5-15.

ROGERO-GARCÍA, J. (2009):“Distribución en España del cuidado formal e informal a las personas de 65 y más años en situación de dependencia”, Revista Española de Salud Pública, vol. 83, n- 3, págs. 393-405.

SHARE-PROJECT (2013a) SHARE Wave 4 Release 1.1.1. [rhttp://dx.doi.org/10.6103/SHARE.w4.111)].

- (2013b): SHARE. Release Guide 2.6.o. Waves 1 \& 2, Mannheim Research Institute for the Economics of Ageing [<http://www.share-project.org/ fileadmin/pdwf_documentation/SHARE_guide_ release_2-6-o.pdf>, consultado el 18-12-2014].

TAPIA, J.; y NIETO, F. J. (1993): "Razón de posibilidades: una propuesta de traducción de la expresión odds ratio", Salud Pública de México, vol. 35, ํㅡ 4, págs.419-424.

TRAVIS, S. S. (1995): “Families and formal networks", en BLIESZNER, R.; y BEDFORD, V. H. (eds.), Handbook of Aging and the Family, Westport, Greenwood Press, págs. 459-473.

VAN GROENOU, M. B., et al. (2006): "Socio-economic status differences in older people's use of informal and formal help: A comparison of four European countries", Ageing and Society, vol. 26, nํㅜ 5, págs. 745-766 [<http://dx.doi.org/10.1017/ S0144686Х06005241)].

VAN HOUTVEN, C. H.; y NORTON, E. C. (2004): "Informal care and health care use of older adults", Journal of Health Economics, vol. 23, nํ 6, págs. 1.159-1.180 [<http://dx.doi.org/10.1016/j. jhealeco.2004.04.008)]. 